\title{
Stability of fractional neutral systems with time varying delay based on delay decomposition approach
}

\author{
Yener Altun \\ Department of Business Administration, Management Faculty, Van Yuzuncu Yil University, 65080, Van, Turkey
}

Received: 10 December 2020, Accepted: 27 December 2020

Published online: 29 December 2020.

\begin{abstract}
The approach in this paper is present asymptotically stability of fractional neutral systems under Riemann-Liouville (RL) derivatives. Some sufficient conditions are devised to such systems based on the Lyapunov method. The difficulty of obtaining a fractional-order Lyapunov functional lies in how to design a positively defined functional $V$ and easily determine whether the fractional derivative of $V$ is less than zero. The method we deal with in this study provides an advantage in terms of directly calculating integer ordered derivatives of Lyapunov functionals. By improving a delay decomposition approach, the stability conditions for the solution of fractional system were established in terms of linear matrix inequalities (LMIs) which can be easily tested. Finally, an example with numerical simulation is given to illustrate the universality and the validity of proposed method.
\end{abstract}

Keywords: Fractional neutral system, asymptotically stability, Lyapunov functional, delay decomposition approach, LMI.

\section{Introduction}

Fractional calculus is a mathematical subject of about 300 years. In the past thirty years, fractional calculus has been found in many various and widespread fields of science and engineering. To describe real world problems, it has been proved that fractional-order calculus is more enough and general than the integer calculus. For this, researchers in the field of mathematics alone do not have a keen interest in fractional calculus. At the same time, fractional systems have received great interest in many scientific areas such as control theory, aircraft stabilization, biophysics, chemical engineering processes, distributed networks, neural networks, nuclear reactors, and population Dynamics and etc. (see [1-23]). For this, interest in the stability analysis of various fractional time delay systems has been increasing quickly in recent years, especially due to its successful applications in common areas of engineering. Therefore, time delay fractional systems and the stability analysis problem for these systems are an important topic both from a theoretical and practical point of view. When the related literature is examined, it is seen that many researchers focus their attention on the stability issue of neutral systems which has a more general class than the delayed type, via different approaches. In this direction, in this study, we improve a delay decomposition approach in fractional neutral systems with time varying delay and obtain novel robust stability criteria.

Motivated and considered by the above discussions, this study searches the asymptotically stability of fractional neutral systems with a delay decomposition approach. We can summarize the main goal of this paper and its contribution to the related literature as follows. First, this paper on the stability of fractional neutral systems with delay decomposition approach is still under development. Therefore, we assert novel stability criteria on the stability of considered fractional system with delay decomposition approach for further improvements. According to the present results in [4, 5, 7, 10, 13], the results of this study are more general. Second, there appears to be very few papers in the literature on the stability of fractional neutral systems with the delay decomposition approach. In this sense, the theoretical results of this paper will contribute to the current related literature. Third, in this paper, some basic inequalities in terms of proof technique are 
used to reduce conservatism and a suitable Lyapunov functional is constructed. Finally, an example with numerical simulation is evaluated to show the universality and the validity of theoretical results.

\section{Problem description}

In this research paper, we consider the following fractional neutral system with time varying delay

$$
{ }_{t_{0}} D_{t}^{q} x(t)-C_{t_{0}} D_{t}^{q} x(t-\tau)=A x(t)+B x(t-\tau(t)), \quad t \geq 0,
$$

with the initial conditions

$$
{ }_{t_{0}} D_{t}^{-(1-q)} x(t)=\varphi(t), \quad t \in[-\tau, 0]
$$

where $0 \leq q \leq 1,{ }_{t_{0}} D_{t}^{q} x($.$) shows a q$ order R-L fractional derivative of $x(),. x(t) \in R^{n}$ is the state vector, $A, B$ and $C \in R^{n \times n}$ are constant matrixes and differentiable function $\tau(t)$ is a variable delay function satisfying for all $t \geq 0$,

$$
0 \leq \tau(t) \leq \tau, \quad \dot{\tau}(t) \leq \delta<1
$$

where $\tau$ and $\delta$ are some positive constants.

Definition 1. The R-L fractional integral and derivative with order $q$ of $x(t)$ are described as follows, respectively ([17])

$$
\begin{aligned}
t_{0} D_{t}^{-q} x(t) & =\frac{1}{\Gamma(q)} \int_{t_{0}}^{t}(t-s)^{q-1} x(s) d s,(q>0), \\
t_{0} D_{t}^{q} x(t) & =\frac{1}{\Gamma(m-q)} \frac{d^{m}}{d t^{m}} \int_{t_{0}}^{t}(t-s)^{m-q-1} x(s) d s,(0 \leq m-1 \leq q<m),
\end{aligned}
$$

where $\Gamma(\cdot)$ states the Gamma function.

Lemma 1. For sufficiently good functions $x(t)$, if $p>q>0$, then the following equality ([9])

$$
{ }_{t_{0}} D_{t}^{q}\left(t_{0} D_{t}^{-p} x(t)\right)={ }_{t_{0}} D_{t}^{q-p} x(t)
$$

holds. In particular, this equality holds if $x(t)$ is integrable.

Lemma 2. Let $x(t) \in R^{n}$ be a vector of differentiable vector. Then, the following inequality holds for any time instant $t \geq 0,([9])$

$$
\frac{1}{2}{ }_{t_{0}} D_{t}^{q}\left(x^{T}(t) \tilde{M} x(t)\right) \leq x^{T}(t) \tilde{M}_{t_{0}} D_{t}^{q} x(t), \quad 0<q<1,
$$

where $\tilde{M} \in R^{n \times n}$ is a constant, square, symmetric and positive semi-definite matrix.

Lemma 3. Given matrices $\Delta_{1}, \Delta_{2}, \Delta_{3}$ where $\Delta_{1}=\Delta_{1}^{T}, \Delta_{3}=\Delta_{3}^{T}$ and $\Delta_{3}>0$. Then $\Delta_{1}+\Delta_{2}^{T} \Delta_{3}^{-1} \Delta_{2}<0$ if and only if ([4])

$$
\left[\begin{array}{cc}
\Delta_{1} & \Delta_{2}^{T} \\
\Delta_{2} & -\Delta_{3}
\end{array}\right]<0 \text { or }\left[\begin{array}{cc}
-\Delta_{3} & \Delta_{2} \\
\Delta_{2}^{T} & -\Delta_{1}
\end{array}\right]<0
$$

\section{Main results}

In this section, by applying delay decomposition approach and constructing an appropriate Lyapunov functional associated with the R-L fractional derivative and fractional integral, we propose a novel stability criterion for the fractional system (1). 
Theorem 1. Let $\|D\|<1$. If $0 \leq \tau(t) \leq \beta \tau$ for given scalars $\beta(0<\beta<1), \tau(>0)$ and $\delta$, the system (1) with (2) is asymptotically stable if there exist matrices $P=P^{T}>0, R=R^{T}>0, H_{i}=H_{i}^{T} \geq 0, U_{i}=U_{i}^{T} \geq 0,(i=1,2,3)$, such that

$$
\widetilde{\Psi}=\left[\begin{array}{cccccc}
\widetilde{\Psi}_{11} & \widetilde{\Psi}_{12} & 0 & 0 & \widetilde{\Psi}_{15} & \widetilde{\Psi}_{16} \\
\widetilde{\Psi}_{12}^{T} & \widetilde{\Psi}_{22} & 0 & 0 & 0 & \widetilde{\Psi}_{26} \\
0 & 0 & \widetilde{\Psi}_{33} & 0 & 0 & 0 \\
0 & 0 & 0 & \widetilde{\Psi}_{44} & 0 & 0 \\
\widetilde{\Psi}_{15}^{T} & 0 & 0 & 0 & \widetilde{\Psi}_{55} & \widetilde{\Psi}_{56} \\
\widetilde{\Psi}_{16}^{T} & \widetilde{\Psi}_{26}^{T} & 0 & 0 & \widetilde{\Psi}_{56}^{T} & \widetilde{\Psi}_{66}
\end{array}\right]<0
$$

with

$$
\begin{aligned}
& \widetilde{\Psi}_{11}=A^{T} P+P A+H_{1}+H_{3}, \widetilde{\Psi}_{12}=P B, \widetilde{\Psi}_{15}=P C, \\
& \widetilde{\Psi}_{16}=A^{T}\left[R+\beta \tau U_{1}+(1-\beta) \tau U_{2}+\beta \tau U_{3}\right], \widetilde{\Psi}_{22}=-(1-\delta) H_{3}, \\
& \widetilde{\Psi}_{26}=B^{T}\left[R+\beta \tau U_{1}+(1-\beta) \tau U_{2}+\beta \tau U_{3}\right], \widetilde{\Psi}_{33}=-H_{2}, \widetilde{\Psi}_{44}=H_{2}-H_{1}, \\
& \widetilde{\Psi}_{55}=-R, \widetilde{\Psi}_{56}=C^{T}\left[R+\beta \tau U_{1}+(1-\beta) \tau U_{2}+\beta \tau U_{3}\right], \\
& \widetilde{\Psi}_{66}=-\left[R+\beta \tau U_{1}+(1-\beta) \tau U_{2}+\beta \tau U_{3}\right] .
\end{aligned}
$$

Proof. Construct the following Lyapunov functional

$$
V\left(x_{t}\right)=\sum_{i=1}^{3} V_{i}\left(x_{t}\right)
$$

where

$$
\begin{aligned}
V_{1}= & t_{0} D_{t}^{q-1}\left(x^{T}(t) P x(t)\right), \\
V_{2}= & \int_{t-\beta \tau}^{t} x^{T}(s) H_{1} x(s) d s+\int_{t-\tau}^{t-\beta \tau} x^{T}(s) H_{2} x(s) d s+\int_{t-\tau(t)}^{t} x^{T}(s) H_{3} x(s) d s+\int_{-\tau}^{0}\left({ }_{t_{0}} D_{t}^{q} x(t+\xi)\right)^{T} R\left(t_{t_{0}} D_{t}^{q} x(t+\xi)\right) d \xi \\
V_{3}= & \int_{-\beta \tau}^{0} \int_{t+\varphi}^{t}\left(t_{t_{0}} D_{\xi}^{q} x(\xi)\right)^{T} U_{1}\left(t_{0} D_{\xi}^{q} x(\xi)\right) d \xi d \varphi+\int_{-\tau}^{-\beta \tau} \int_{t+\varphi}^{t}\left(t_{0} D_{\xi}^{q} x(\xi)\right)^{T} U_{2}\left(t_{t_{0}} D_{\xi}^{q} x(\xi)\right) d \xi d \varphi \\
& +\int_{-\tau(t)}^{0} \int_{t+\varphi}^{t}\left(t_{0} D_{\xi}^{q} x(\xi)\right)^{T} U_{3}\left(t_{0} D_{\xi}^{q} x(\xi)\right) d \xi d \varphi .
\end{aligned}
$$

From Definition 1., we know that, $V_{1}, V_{2}$ and $V_{3}$ are positive definite functional. By Lemma 1 and Lemma 2, calculating time derivative $V\left(x_{t}\right)$ along the trajectory of system (1), we obtain

$$
\dot{V}\left(x_{t}\right)=\sum_{i=1}^{3} \dot{V}_{i}\left(x_{t}\right)
$$

where

$$
\begin{aligned}
\dot{V}_{1}\left(x_{t}\right) & ={ }_{t_{0}} D_{t}^{q}\left(x^{T}(t) P x(t)\right) \leq 2 x^{T}(t) P_{t_{0}} D_{t}^{q}(x(t)) \\
& =2 x^{T}(t) P\left[A x(t)+B x(t-\tau(t))+C_{t_{0}} D_{t}^{q} x(t-\tau)\right] \\
& =x^{T}(t)\left(P A+A^{T} P\right) x(t)+2 x^{T}(t) P B x(t-\tau(t))+2 x^{T}(t) P C_{t_{0}} D_{t}^{q} x(t-\tau)
\end{aligned}
$$




$$
\begin{gathered}
\dot{V}_{2}\left(x_{t}\right)=x^{T}(t)\left(H_{1}+H_{3}\right) x(t)-x^{T}(t-\tau(t))(1-\dot{\tau}(t)) H_{3} x(t-\tau(t)) \\
+x^{T}(t-\beta \tau)\left(H_{2}-H_{1}\right) x(t-\beta \tau)-x^{T}(t-\tau) H_{2} x(t-\tau) \\
\quad+\left({ }_{t_{0}} D_{t}^{q} x(t)\right)^{T} R\left({ }_{t_{0}} D_{t}^{q} x(t)\right)-\left(t_{0} D_{t}^{q} x(t-\tau)\right)^{T} R\left(t_{t_{0}} D_{t}^{q} x(t-\tau)\right) \\
\leq x^{T}(t)\left(H_{1}+H_{3}\right) x(t)-x^{T}(t-\tau(t))(1-\delta) H_{3} x(t-\tau(t)) \\
\quad+x^{T}(t-\beta \tau)\left(H_{2}-H_{1}\right) x(t-\beta \tau)-x^{T}(t-\tau) H_{2} x(t-\tau) \\
\left.\quad+\left(t_{0} D_{t}^{q} x(t)\right)^{T} R\left(t_{0} D_{t}^{q} x(t)\right)-\left(t_{0} D_{t}^{q} x(t-\tau)\right)\right)^{T} R\left(t_{t_{0}} D_{t}^{q} x(t-\tau)\right) \\
\dot{V}_{3}\left(x_{t}\right)=\left(t_{t_{0}} D_{t}^{q} x(t)\right)^{T}\left(\beta \tau U_{1}+(1-\beta) \tau U_{2}+\tau(t) U_{3}\right)_{t_{0}} D_{t}^{q} x(t) \\
-\int_{t-\beta \tau}^{t}\left({ }_{t_{0}} D_{s}^{q} x(s)\right)^{T} U_{1}\left(t_{0} D_{s}^{q} x(s)\right) d s-\int_{t-\tau}^{t-\beta \tau}\left({ }_{t_{0}} D_{s}^{q} x(s)\right)^{T} U_{2}\left(t_{t_{0}} D_{s}^{q} x(s)\right) d s \\
-(1-\dot{\tau}(t)) \int_{t-\tau(t)}^{t}\left(t_{t_{0}} D_{s}^{q} x(s)\right)^{T} U_{3}\left({ }_{t_{0}} D_{s}^{q} x(s)\right) d s \\
\leq\left(t_{t_{0}} D_{t}^{q} x(t)\right)^{T}\left(\beta \tau U_{1}+(1-\beta) \tau U_{2}+\beta \tau U_{3}\right)_{t_{0}} D_{t}^{q} x(t) \\
-\int_{t-\beta \tau}^{t}\left({ }_{t_{0}} D_{s}^{q} x(s)\right)^{T} U_{1}\left({ }_{t_{0}} D_{s}^{q} x(s)\right) d s-\int_{t-\tau}^{t-\beta \tau}\left({ }_{t_{0}} D_{s}^{q} x(s)\right)^{T} U_{2}\left(t_{t_{0}} D_{s}^{q} x(s)\right) d s \\
-(1-\delta) \int_{t-\tau(t)}^{t}\left({ }_{t_{0}} D_{s}^{q} x(s)\right)^{T} U_{3}\left({ }_{t_{0}} D_{s}^{q} x(s)\right) d s .
\end{gathered}
$$

From (7) and (8), the operator for term $\left({ }_{t_{0}} D_{t}^{q} x(t)\right)^{T}\left[R+\beta \tau U_{1}+(1-\beta) \tau U_{2}+\beta \tau U_{3}\right]_{t_{0}} D_{t}^{q} x(t)$ is as follows:

$$
\begin{aligned}
& \left(t_{0} D_{t}^{q} x(t)\right)^{T}\left[R+\beta \tau U_{1}+(1-\beta) \tau U_{2}+\beta \tau U_{3}\right]_{t_{0}} D_{t}^{q} x(t) \\
= & {\left[A x(t)+B x(t-\tau(t))+C_{t_{0}} D_{t}^{q} x(t-\tau)\right]^{T}\left[R+\beta \tau U_{1}+(1-\beta) \tau U_{2}+\beta \tau U_{3}\right] } \\
& \times\left[A x(t)+B x(t-\tau(t))+C_{t_{0}} D_{t}^{q} x(t-\tau)\right] \\
= & x^{T}(t) A^{T}\left[R+\beta \tau U_{1}+(1-\beta) \tau U_{2}+\beta \tau U_{3}\right] A x(t) \\
& +x^{T}(t) A^{T}\left[R+\beta \tau U_{1}+(1-\beta) \tau U_{2}+\beta \tau U_{3}\right] B x(t-\tau(t)) \\
& +x^{T}(t) A^{T}\left[R+\beta \tau U_{1}+(1-\beta) \tau U_{2}+\beta \tau U_{3}\right] C_{t_{0}} D_{t}^{q} x(t-\tau) \\
& +x^{T}(t-\tau(t)) B^{T}\left[R+\beta \tau U_{1}+(1-\beta) \tau U_{2}+\beta \tau U_{3}\right] A x(t) \\
& +x^{T}(t-\tau(t)) B^{T}\left[R+\beta \tau U_{1}+(1-\beta) \tau U_{2}+\beta \tau U_{3}\right] B x(t-\tau(t)) \\
& +x^{T}(t-\tau(t)) B^{T}\left[R+\beta \tau U_{1}+(1-\beta) \tau U_{2}+\beta \tau U_{3}\right] C_{t_{0}} D_{t}^{q} x(t-\tau) \\
& +\left(_{t_{0}} D_{t}^{q} x(t-\tau)\right)^{T} C^{T}\left[R+\beta \tau U_{1}+(1-\beta) \tau U_{2}+\beta \tau U_{3}\right] A x(t) \\
& +\left(_{t_{0}} D_{t}^{q} x(t-\tau)\right)^{T} C^{T}\left[R+\beta \tau U_{1}+(1-\beta) \tau U_{2}+\beta \tau U_{3}\right] B x(t-\tau(t)) \\
& \left.+{ }_{t_{0}} D_{t}^{q} x(t-\tau)\right)^{T} C^{T}\left[R+\beta \tau U_{1}+(1-\beta) \tau U_{2}+\beta \tau U_{3}\right] C_{t_{0}} D_{t}^{q} x(t-\tau) .
\end{aligned}
$$

Combining (6)-(9) yields that

$$
\begin{aligned}
\dot{V}\left(x_{t}\right) & \leq \xi^{T}(t) \Sigma \xi(t)-\int_{t-\beta \tau}^{t}\left({ }_{t_{0}} D_{s}^{q} x(s)\right)^{T} U_{1}\left(t_{t_{0}} D_{s}^{q} x(s)\right) d s-\int_{t-\tau}^{t-\beta \tau}\left({ }_{t_{0}} D_{s}^{q} x(s)\right)^{T} U_{2}\left(t_{t_{0}} D_{s}^{q} x(s)\right) d s \\
& -\int_{t-\tau(t)}^{t}\left(t_{t_{0}} D_{s}^{q} x(s)\right)^{T}\left((1-\delta) U_{3}\right)\left(t_{t_{0}} D_{s}^{q} x(s)\right) d s
\end{aligned}
$$

where

$$
\left.\xi^{T}(t)=\left[x^{T}(t) x^{T}(t-\tau(t)) x^{T}(t-\tau)\right) x^{T}(t-\beta \tau)\left({ }_{t_{0}} D_{t}^{q} x(t-\tau)\right)^{T}\right]
$$


and

$$
\Sigma=\left[\begin{array}{ccccc}
\Sigma_{11} & \Sigma_{12} & 0 & 0 & \Sigma_{15} \\
\Sigma_{12}^{T} & \Sigma_{22} & 0 & 0 & \Sigma_{25} \\
0 & 0 & \Sigma_{33} & 0 & 0 \\
0 & 0 & 0 & \Sigma_{44} & 0 \\
\Sigma_{15}^{T} & \Sigma_{25}^{T} & 0 & 0 & \Sigma_{55}
\end{array}\right],
$$

with

$$
\begin{aligned}
& \Sigma_{11}=A^{T} P+P A+H_{1}+H_{3}+A^{T}\left[R+\beta \tau U_{1}+(1-\beta) \tau U_{2}+\beta \tau U_{3}\right] A, \\
& \Sigma_{12}=P B+A^{T}\left[R+\beta \tau U_{1}+(1-\beta) \tau U_{2}+\beta \tau U_{3}\right] B, \\
& \Sigma_{15}=P C+A^{T}\left[R+\beta \tau U_{1}+(1-\beta) \tau U_{2}+\beta \tau U_{3}\right] C, \\
& \Sigma_{22}=B^{T}\left[R+\beta \tau U_{1}+(1-\beta) \tau U_{2}+\beta \tau U_{3}\right] B-(1-\delta) H_{3}, \\
& \Sigma_{25}=B^{T}\left[R+\beta \tau U_{1}+(1-\beta) \tau U_{2}+\beta \tau U_{3}\right] C, \\
& \Sigma_{33}=-H_{2}, \\
& \Sigma_{44}=H_{2}-H_{1}, \\
& \Sigma_{55}=-R+C^{T}\left[R+\beta \tau U_{1}+(1-\beta) \tau U_{2}+\beta \tau U_{3}\right] C .
\end{aligned}
$$

For $\xi(t) \neq 0$ and $0 \leq \tau(t) \leq \beta \tau$, if $\Sigma<0, \dot{V}\left(x_{t}\right)$ is negative-definite. From Lemma 3, if condition (3) is met, system (1) is asymptotically stable.

Theorem 2. Let $\|D\|<1$. If $\beta \tau \leq \tau(t) \leq \tau$ for given scalars $\beta(0<\beta<1), \tau(>0)$ and $\delta$, the system (1) with (2) is asymptotically stable if there exist matrices $P=P^{T}>0, R=R^{T}>0, H_{i}=H_{i}^{T} \geq 0, U_{i}=U_{i}^{T} \geq 0,(i=1,2,3)$, such that

$$
\widetilde{\Pi}=\left[\begin{array}{cccccc}
\widetilde{\Pi}_{11} & \widetilde{\Pi}_{12} & 0 & 0 & \widetilde{\Pi}_{15} & \widetilde{\Pi}_{16} \\
\widetilde{\Pi}_{12}^{T} & \widetilde{\Pi}_{22} & 0 & 0 & 0 & \widetilde{\Pi}_{26} \\
0 & 0 & \widetilde{\Pi}_{33} & 0 & 0 & 0 \\
0 & 0 & 0 & \widetilde{\Pi}_{44} & 0 & 0 \\
\widetilde{\Pi}_{15}^{T} & 0 & 0 & 0 & \widetilde{\Pi}_{55} & \widetilde{\Pi}_{56} \\
\widetilde{\Pi}_{16}^{T} & \widetilde{\Pi}_{26}^{T} & 0 & 0 & \widetilde{\Pi}_{56}^{T} & \widetilde{\Pi}_{66}
\end{array}\right]<0 .
$$

with

$$
\begin{aligned}
\widetilde{\Pi}_{11} & =A^{T} P+P A+H_{1}+H_{3}, \widetilde{\Pi}_{12}=P B, \widetilde{\Pi}_{15}=P C, \\
\widetilde{\Pi}_{16} & =A^{T}\left[R+\beta \tau U_{1}+(1-\beta) \tau U_{2}+\tau U_{3}\right], \widetilde{\Pi}_{22}=-(1-\delta) H_{3}, \\
\widetilde{\Pi}_{26} & =B^{T}\left[R+\beta \tau U_{1}+(1-\beta) \tau U_{2}+\tau U_{3}\right], \widetilde{\Pi}_{33}=-H_{2}, \widetilde{\Pi}_{44}=H_{2}-H_{1}, \\
\widetilde{\Pi}_{55} & =-R, \widetilde{\Pi}_{56}=C^{T}\left[R+\beta \tau U_{1}+(1-\beta) \tau U_{2}+\tau U_{3}\right], \\
\widetilde{\Pi}_{66} & =-\left[R+\beta \tau U_{1}+(1-\beta) \tau U_{2}+\tau U_{3}\right] .
\end{aligned}
$$

Proof. Consider the Lyapunov functional defined by (4) in Theorem 1 for the proof of this theorem. For $\beta \tau \leq \tau(t) \leq \tau$, calculating time derivative $V\left(x_{t}\right)$ along the trajectory of system (1), we have

$$
\begin{aligned}
\dot{V}\left(x_{t}\right) \leq & \lambda^{T}(t) \Omega \lambda(t)-\int_{t-\beta \tau}^{t}\left(t_{0} D_{s}^{q} x(s)\right)^{T} U_{1}\left({ }_{t_{0}} D_{s}^{q} x(s)\right) d s \\
& -\int_{t-\tau}^{t-\beta \tau}\left(t_{t_{0}} D_{s}^{q} x(s)\right)^{T} U_{2}\left({ }_{t_{0}} D_{s}^{q} x(s)\right) d s-\int_{t-\tau(t)}^{t}\left({ }_{t_{0}} D_{s}^{q} x(s)\right)^{T}\left((1-\delta) U_{3}\right)\left({ }_{t_{0}} D_{s}^{q} x(s)\right) d s
\end{aligned}
$$


where

$$
\left.\lambda^{T}(t)=\left[x^{T}(t) x^{T}(t-\tau(t)) x^{T}(t-\tau)\right) x^{T}(t-\beta \tau)\left({ }_{t_{0}} D_{t}^{q} x(t-\tau)\right)^{T}\right]
$$

and

$$
\Omega=\left[\begin{array}{ccccc}
\Omega_{11} & \Omega_{12} & 0 & 0 & \Omega_{15} \\
\Omega_{12}^{T} & \Omega_{22} & 0 & 0 & \Omega_{25} \\
0 & 0 & \Omega_{33} & 0 & 0 \\
0 & 0 & 0 & \Omega_{44} & 0 \\
\Omega_{15}^{T} & \Omega_{25}^{T} & 0 & 0 & \Omega_{55}
\end{array}\right]
$$

with

$$
\begin{aligned}
& \Omega_{11}=A^{T} P+P A+H_{1}+H_{3}+A^{T}\left[R+\beta \tau U_{1}+(1-\beta) \tau U_{2}+\tau U_{3}\right] A, \\
& \Omega_{12}=P B+A^{T}\left[R+\beta \tau U_{1}+(1-\beta) \tau U_{2}+\tau U_{3}\right] B, \\
& \Omega_{15}=P C+A^{T}\left[R+\beta \tau U_{1}+(1-\beta) \tau U_{2}+\tau U_{3}\right] C, \\
& \Omega_{22}=B^{T}\left[R+\beta \tau U_{1}+(1-\beta) \tau U_{2}+\tau U_{3}\right] B-(1-\delta) H_{3}, \\
& \Omega_{25}=B^{T}\left[R+\beta \tau U_{1}+(1-\beta) \tau U_{2}+\tau U_{3}\right] C, \Omega_{33}=-H_{2}, \\
& \Omega_{44}=H_{2}-H_{1}, \Omega_{55}=-R+C^{T}\left[R+\tau U_{1}+(1-\beta) \tau U_{2}+\tau U_{3}\right] C .
\end{aligned}
$$

For $\lambda(t) \neq 0$ and $\beta \tau \leq \tau(t) \leq \tau$, if $\Omega<0, \dot{V}\left(x_{t}\right)$ is negative-definite. From Lemma 3, if condition (10) is met, system (1) is asymptotically stable.

\section{Numerical example}

In this section, we give an example with numerical simulation to show the advantages and validity of our results.

Example 1. Consider the following fractional neutral system for $t \geq 0$,

$$
{ }_{t_{0}} D_{t}^{q} x(t)-\left[\begin{array}{cc}
0.4 & 0 \\
0 & 0.1
\end{array}\right]{ }_{t_{0}} D_{t}^{q} x(t-\tau)=\left[\begin{array}{cc}
-2.5 & -0.2 \\
0.1 & -4.2
\end{array}\right] x(t)+\left[\begin{array}{cc}
-0.1 & 0.1 \\
-0.2 & 0.1
\end{array}\right] x(t-\tau(t)),
$$

where $x(t)=\left[x_{1}(t) x_{2}(t)\right]^{T}$ and

$$
0 \leq \tau(t)=0.4 \sin ^{2}(t) \leq 0.4=\tau
$$

Applying Theorem 1 and Theorem 2 to above system (11) with (12), for $\beta=0.05$, let us choose

$$
\begin{gathered}
P=\left[\begin{array}{cc}
15 & 0 \\
0 & 18
\end{array}\right], R=\left[\begin{array}{ll}
4 & 1 \\
1 & 2
\end{array}\right], \\
H_{1}=\left[\begin{array}{cc}
18.34 & -0.04 \\
-0.04 & 5,42
\end{array}\right], H_{2}=\left[\begin{array}{cc}
12.26 & -0.36 \\
-0.36 & 0,48
\end{array}\right], H_{3}=\left[\begin{array}{cc}
1.22 & -0.14 \\
-0.14 & 0.02
\end{array}\right], \\
U_{1}=\left[\begin{array}{cc}
24.02 & 4.04 \\
4.04 & 14.18
\end{array}\right], U_{2}=\left[\begin{array}{cc}
2.62 & -0.09 \\
-0.09 & 1.12
\end{array}\right], U_{3}=\left[\begin{array}{cc}
0.12 & -0.01 \\
-0.01 & 0.02
\end{array}\right] .
\end{gathered}
$$

Thus, considering above assumptions and taking advantage of MATLAB-Simulink the all eigenvalues of the LMIs defined by (3) and (10) are $\lambda_{\max }(\widetilde{\Psi}) \leq-0.0991$ and $\lambda_{\max }(\widetilde{\Pi}) \leq-0.1163$, respectively. This ensures that system (11) with (12) is asymptotically stable. 


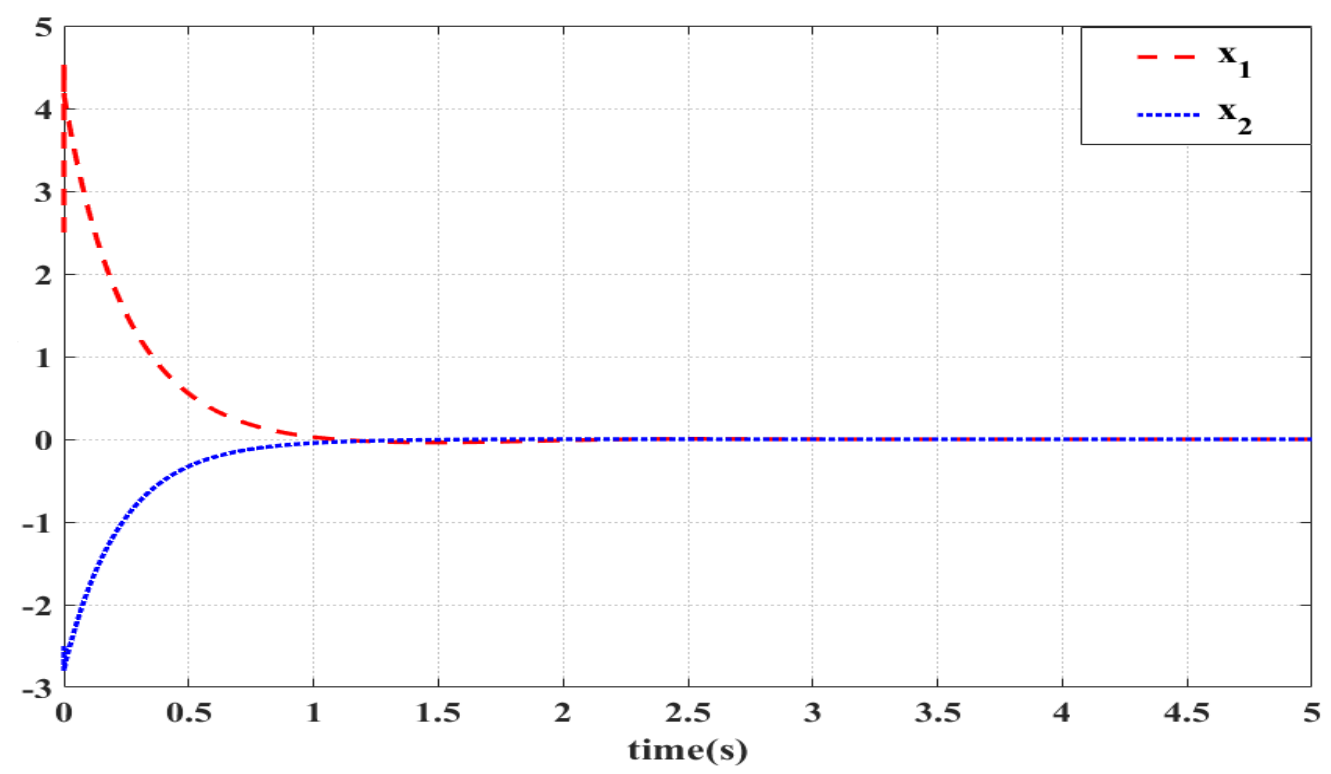

Fig. 1: The state response of system (11) for (12).

\section{Conclusion}

This paper studies the problem of asymptotically stability for fractional neutral systems under R-L derivatives. By developing a delay decomposition approach, some novel stability criteria are obtained in terms of linear matrix inequalities (LMIs) based on the Lyapunov method. An example with numerical simulation is presented to demonstrate the universality and the validity of the considered system. Finally, the theoretical results of this paper contribute to generalize and expand the present ones in the literature.

\section{Competing interests}

The authors declare that they have no competing interests.

\section{Authors' contributions}

All authors have contributed to all parts of the article. All authors read and approved the final manuscript.

\section{References}

[1] Agarwal, R.P., Benchohra, M. and Hamani, S. A survey on existence results for boundary value problems of nonlinear fractional differential equations and inclusions. Acta Appl. Math., 109(3), 973-1033, 2010.

[2] Agarwal R.P. and Grace S.R. Asymptotic stability of differential systems of neutral type. Appl. Math. Lett., 13(8), 15-19, 2000.

[3] Altun Y. Further results on the asymptotic stability of Riemann-Liouville fractional neutral systems with variable delays. Adv. Difference Equ., 437, 1-13, 2019.

[4] Altun, Y. Improved results on the stability analysis of linear neutral systems with delay decay approach. Math Meth Appl Sci., 43, 1467-1483, 2020. (2014). 
[5] Altun Y. New Results on the Exponential Stability of Class Neural Networks with Time-Varying Lags. BEU Journal of Science, 8, 443-450, 2019.

[6] Boyd, B. Ghoui, L.E. Feron, E. and Balakrishnan, V. Linear matrix inequalities in system and control theory. Philadephia: SIAM. 1994.

[7] Chen, L. He, Y. and Chai, Y. Wu, R. New results on stability stabilization of a class of nonlinear fractional-order systems. Nonlinear Dynam., 75(4), 633-641, 2014.

[8] Deng, J. and Deng, Z. Existence of solutions of initial value problems for nonlinear fractional differential equations. Appl. Math. Lett., 32, 6-12, 2014.

[9] Kilbas, A.A. Srivastava, H.M. and Trujillo, J.J. Theory and Application of Fractional Differential Equations. Elsevier, New York, 2006.

[10] Li, H. Zhou, S. and Li, H. Asymptotic stability analysis of fractional-order neutral systems with time delay. Adv. Difference Equ., 2015, 325-335, 2015.

[11] Liu, K. and Jiang, W. Stability of fractional neutral systems. Adv. Differ. Equ., 2014, 1-9, 2014.

[12] Liu, P.L. A delay decomposition approach to stability analysis of neutral systems with time-varying delay. Appl. Math Model., 37, 5013-26, 2013.

[13] Liu, S. Wu, X. Zhang, Y.J. and Yang, R. Asymptotical stability of Riemann-iouville fractional neutral systems. Appl. Math. Lett., 69,168-173, 2017.

[14] Liu, S. Zhou, X.F. Li, X. and Jiang, W. Stability of fractional nonlinear singular systems its applications in synchronization of complex dynamical networks. Nonlinear Dynam., 84, 2377-2385, 2016.

[15] Lu, J.G. and Chen, G. Robust stability and stabilization of fractional-order interval systems: An LMI approach. IEEE Trans. Automat. Control, 54(6), 1294-1299, 2009.

[16] Lu, Y.F. Wu, R.C. and Qin, Z.Q. Asymptotic stability of nonlinear fractional neutral singular systems. J. Appl. Math. Comput., 45, 351-364, 2014.

[17] Podlubny, I. Fractional Differential Equations. Academic Press., New York, USA, 1999.

[18] Qian, D. Li, C. Agarwal, R.P. and Wong P.J.Y. Stability analysis of fractional differential system with Riemann-Liouville derivative. Math. Comput. Model., 52, 862-874, 2010.

[19] Sabatier, J. Moze, M. and Farges, C. LMI stability conditions for fractional order systems. Comput. Math. Appl., 59(5), 1594-1609, 2010.

[20] Wang, J. Lv, L. and Zhou, Y. New concepts and results in stability of fractional differential equations. Commun. Nonlinear Sci., 17(6), 2530-2538, 2012.

[21] Zhang, H. Ye, R. Cao, J. and Alsaedi, A. Delay-independent stability of Riemann-Liouville fractional neutral-type delayed neural networks. Neural Proces. Lett., 47, 427-442, 2018.

[22] Zhang, H. Ye, R. Liu, S. Cao, J. Alsaedi, A. and Li, X. LMI-based approach to stability analysis for fractional-order neural networks with discrete and distributed delays. Int. J. Syst. Sci., 49, 537-545, 2018.

[23] Zhou, X. F. Hu, L.G. Liu, S. and Jiang, W. Stability criterion for a class of nonlinear fractional differential systems. Appl. Math. Lett., 28, 25-29, 2014. 